\title{
Rechtsprechung
}

\section{Einsicht in die Unterlagen der Beratenden Kommission für die Rückgabe NS- verfolgungsbedingt entzogener Kulturgüter}

Verwaltungsgericht Magdeburg, Urteil vom 31. März 2015 - 6 A 81/15

Die Beratende Kommission für die Rückgabe NS-verfolgungsbedingt entzogener Kulturgüter nimmt keine öf-
fentlich-rechtlichen Verwaltungsaufgaben wahr und ist deshalb gemäß $§ 1$ Abs. 1 Informationszugangsgesetz (IFG) nicht verpflichtet, Dritten Einsicht in ihre Akten zu gewähren. (Amtlicher Leitsatz)

\section{Tatbestand}

- Der Kläger begehrt Einsicht in alle Unterlagen der Beratenden Kommission für die Rückgabe NS-verfolgungsbedingt entzogener Kulturgüter zu einem Rückerstattungsverfahren von NSRaubkunst.

Der Kläger war der Rechtsanwalt von Herrn P. S., dem Sohn und Rechtsnachfolger des jüdischen Zahnarztes Dr. H. S. Dieser hatte seit 1896 eine umfangreiche und wertvolle Plakatsammlung zusammengetragen, welche ihm 1938 im Auftrag des Reichspropagandaministeriums weggenommen wurde. Wegen der nationalsozialistischen Judenverfolgung verließ Dr. S. Deutschland Ende 1938 und emigrierte in die USA. Nach dem Krieg war die Sammlung zunächst verschollen. Später stellte sich heraus, dass sich Teile der Sammlung im Museum für Deutsche Geschichte in Ost-Berlin befanden. Zuletzt befand sich die Plakatsammlung im Besitz des Deutschen Historischen Museums, einer Stiftung des öffentlichen Rechts unter alleiniger Trägerschaft des Bundes.

Im Jahr 2005 verlangte Herr P. S. die Herausgabe der Sammlung. Nach der Prüfung der Restitutionsunterlagen lehnte das Deutsche Historische Museum eine Rückgabe der Sammlung ab. Nachdem die Parteien keine Einigung erzielen konnten, verständigten sie sich darauf, den Fall der Beratenden Kommission für die Rückgabe NS-verfolgungsbedingt entzogener Kulturgüter (im Folgenden: Beratende Kommission) vorzulegen. Hierbei handelt es sich um ein mit hochrangigen, ehrenamtlich tätigen Persönlichkeiten aus Wissenschaft und Gesellschaft besetztes Gremium, das bei Meinungsverschiedenheiten zwischen den heutigen Besitzern und den ehemaligen Eigentümern von Kulturgütern oder deren Erben vermitteln kann, wenn dies von beiden Seiten gewünscht wird. Im vorliegenden Fall gab die Beratende Kommission im Januar 2007 die Empfehlung ab, angesichts des deutlich zum Ausdruck gebrachten Willens des Sammlers Dr. S. die Sammlung im Deutschen Historischen Museum zu belassen.

Der Streit war damit nicht beigelegt. Herr P. S. strengte vor dem Landgericht Berlin ein Musterverfahren gerichtet auf Herausgabe des Plakats "Die Dogge“ an, das er gewann. Die hiergegen eingelegte Berufung des Deutschen Historischen Muse- ums wurde vom Bundesgerichtshof zurückgewiesen. Auch die Anschlussrevision des Deutschen Historischen Museums, mit der dieses das fehlende Eigentum des Herrn P. S. an der Plakatsammlung festgestellt wissen wollte, wurde zurückgewiesen. Dr. S. sei zu Lebzeiten Eigentümer der Sammlung geblieben. Nach seinem Tod sei das Eigentum im Wege der Erbfolge zunächst auf seine Ehefrau und anschließend auf Herrn P. S. übergegangen (vgl. BGH, Urteil v. 16. März 2012 - V ZR 279/10, NJW 2012, S. $1796<1797$ ff. $>$ ).

Mit Schreiben vom 24. Juni 2013 beantragte der Kläger im Namen von Herrn P. S. bei der Koordinierungsstelle für Kulturgutverluste Akteneinsicht in die Unterlagen der Beratenden Kommission zum Fall S. Die Koordinierungsstelle für Kulturgutverluste (im Folgenden: Koordinierungsstelle) ist eine von der Bundesrepublik Deutschland und den Ländern getragene Einrichtung in Form einer Arbeitsgruppe des Kultusministeriums des Landes Sachsen-Anhalt, zu deren Aufgaben u.a. die Funktion der Geschäftsstelle der Beratenden Kommission gehört (§ 1 Abs. 3 c der Gemeinsamen Vereinbarung über die Koordinierungsstelle Magdeburg 2010-2016). Der Kläger begründete das Akteneinsichtsgesuch mit dem Hinweis, dass das Restitutionsverfahren abgeschlossen sei; Geheimhaltungsinteressen könnten daher nicht mehr bestehen.

Mit Schreiben vom 23. September 2013 lehnte die Koordinierungsstelle den Antrag auf Akteneinsicht im Namen und Auftrag der Vorsitzenden der Beratenden Kommission, Frau Professor Dr. L., ab. Zur Begründung hieß es, die Beratende Kommission sei keine Bundesbehörde im Sinne von § 1 Informationsfreiheitsgesetz (IFG), da sie weder Aufgaben der öffentlichen Verwaltung wahrnehme noch für sonstige Bundesorgane und -einrichtungen öffentlich-rechtliche Verwaltungsaufgaben ausführe. Bei der Beratenden Kommission handele es sich um ein reines Beratungsgremium von ehrenamtlich tätigen hochrangigen Personen aus der Wissenschaft und dem öffentlichen Leben, die unverbindliche Empfehlungen gegenüber Einrichtungen und Personen aussprächen. Diese Empfehlungen basierten auf ethischen Abwägungsentscheidungen, denen ein moralisches Räsonnement zu Grunde liege. In diesem Zusammenhang erstatteten die Berichterstatter der Kommission nur mündliche Berichte aus den von den Verfahrensbeteiligten eingereichten Akten, die jeweils auch der Gegenseite zugestellt 
würden. Die dabei hin und wieder schriftlich vorliegenden Berichte beschränkten sich zumeist auf eine summarische Wiedergabe des Akteninhalts und gäben keine Auskunft über die - spätere - moralische Reflektion der Kommission hinsichtlich deren Empfehlung. Selbst in den Protokollen der Kommissionssitzungen fänden sich keine Auskünfte hierzu. Die Kommission habe sich bereits anlässlich ihrer Gründung im Jahr 2003 darauf verständigt, zu ihren Sitzungen ausschließlich Verlaufsprotokolle durch die Geschäftsstelle fertigen zu lassen. Überdies bestehe auch das hohe persönliche Interesse aller Kommissionsmitglieder, dass deren Unabhängigkeit in den Beratungen nicht dadurch beeinträchtigt werde, dass Unterlagen der Beratenden Kommission - gleich welcher Art - öffentlich zugänglich gemacht werden. Die Arbeit der Beratenden Kommission sei nur möglich, wenn deren Tätigkeit vertraulich bleibe.

Mit Schreiben vom 14. Oktober 2013 wiederholte und bekräftige der Kläger den Antrag auf Akteneinsicht. Die Beratende Kommission sei eine öffentliche Einrichtung und nehme im Sinne der einschlägigen Gesetze Aufgaben der öffentlichen Verwaltung wahr. Dies zeige sich schon daran, dass sie von der öffentlichen Hand vollständig bezahlt werde. Es werde einem rechtsmittelfähigen Bescheid entgegengesehen oder um Mitteilung gebeten, ob das Schreiben der Koordinierungsstelle vom 23. September 2013 als Bescheid anzusehen sei. In diesem Fall würde Verpflichtungsklage erhoben. Mit Schreiben vom 16. Dezember 2013 teilte die Koordinierungsstelle, wiederum im Namen und Auftrag der Vorsitzenden der Beratenden Kommission, dem Kläger mit, dass die Beratende Kommission, deren Geschäftsstelle und die Koordinierungsstelle rechtlich unselbständig bzw. keine Behörden seien und deshalb rechtsmittelfähigen Bescheid nicht erlassen könnten. Mit einer Klage wäre daher das Schreiben vom 23. September 2013 anzugreifen.

\section{Am 29. Januar 2014 hat der Kläger Klage erhoben.}

Der Kläger ist der Ansicht, dass es sich bei der Koordinierungsstelle um eine Behörde im Sinne von § 1 Abs. 1 S. 1 Informationszugangsgesetz Sachsen-Anhalt (IZG LSA) handele; diese sei gemäß § 7 Abs. 1 IZG LSA auskunftsverpflichtet. Der Verwaltungsbegriff sei weit auszulegen; darunter fielen auch beratende Tätigkeiten. Die Koordinierungsstelle werde auch im bundesdeutschen Interesse tätig, da sie u.a. das elektronische Verzeichnis national wertvollen Kulturgutes betreue. Im vorliegenden Zusammenhang habe die Koordinierungsstelle dem Deutschen Historischen Museum weitreichende Empfehlungen erteilt und ein gemeinsames Vorgehen vorbereitet. Zu diesem Zweck seien Gutachten u.a. zu erwarteten politischen Auswirkungen des Rechtsstreits zwischen Herrn P. S. und dem Deutschen Historischen Museum eingeholt, ausgewertet und weitergeleitet worden. Auch jeglicher Schriftverkehr des Herrn P. S. mit der Koordinierungsstelle sei umgehend an das Kultusministerium des Landes Sachsen-Anhalt sowie an den Bundesminister für Kultur und Medien weitergeleitet worden. Das Ausmaß dieses umfassenden Informationsaustauschs sei bislang nicht offengelegt worden. Ausschlussgründe nach §§ 3, 4 IZG
LSA stünden dem Informationsanspruch nicht entgegen. Insbesondere werde der Kernbereich exekutiver Eigenverantwortung nicht berührt. Es gehe um eine Grundsatzklärung, ob die Beratende Kommission und deren Geschäftsstelle im rechtsfreien Raum agierten. Unabhängig von der innerorganisatorischen Zuständigkeits- und Aufgabenverteilung müsse jedenfalls einer der Beklagten zur Gewährung von Akteneinsicht verpflichtet sein.

Der Kläger beantragt,

die Beklagten unter Aufhebung des Bescheids vom 23. September 2013 und des Widerspruchsbescheids vom 16. Dezember 2013 zu verpflichten, Akteneinsicht in alle Unterlagen der Beratenden Kommission zum Fall P. S. gegen Deutsches Historisches Museum wegen Rückgabe der Plakatsammlung S. zu erteilen.

Die Beklagten beantragen,

die Klage abzuweisen.

Zur Begründung heißt es, die Beklagte zu 1. sei zur Gewährung der begehrten Akteneinsicht nicht befugt. Die rechtliche Verfügungsbefugnis liege bei der Beratenden Kommission, bei der es sich aber um keine Behörde handele, da sie vollkommen unabhängig agiere und Verwaltungsentscheidungen weder selbst treffe noch vorbereite. Die Beratende Kommission sei ein reines Beratungsgremium, das rechtlich unverbindliche Empfehlungen auf Grundlage ethischer Abwägungsentscheidungen ausspreche.

Während des gerichtlichen Verfahrens haben Bund, Länder und kommunale Spitzenverbände mit Wirkung vom 1. Januar 2015 die Stiftung "Deutsches Zentrum Kulturgutverluste" in der Form einer rechtsfähigen Stiftung des bürgerlichen Rechts gegründet, die u.a. die Aufgaben der Koordinierungsstelle fortführt.

Wegen der weiteren Einzelheiten des Sach- und Streitstandes und des Vorbringens der Beteiligten im Übrigen wird auf die Gerichtsakte verwiesen. Diese Unterlagen sind Gegenstand der mündlichen Verhandlung gewesen.

\section{Entscheidungsgründe}

Die Klage hat keinen Erfolg.

Soweit der Kläger eine Verpflichtung des Landes SachsenAnhalt begehrt, ist der Klageantrag dahingehend auszulegen, dass sich die Klage gemäß § 78 Abs. 1 Nr. 2 VwGO iVm § 8 AG VwGO LSA gegen das Kultusministerium des Landes SachsenAnhalt richtet. Bei der Koordinierungsstelle handelt es sich um eine unselbständige Arbeitsgruppe des Kultusministeriums 
Sachsen-Anhalt (vgl. OVG LSA, Urteil v. 23. Oktober 2013 - 3 L 84/12, juris, Rn. 29), dem die Handlungen der Koordinierungsstelle damit zuzurechnen sind.

Die Klage richtet sich weiterhin (u.a.) gegen das Kultusministerium Sachsen-Anhalt. Dass die Aufgaben der Koordinierungsstelle inzwischen von einer rechtsfähigen Stiftung des bürgerlichen Rechts fortgeführt werden, hat keinen gesetzlichen Parteiwechsel auf Beklagtenseite zur Folge. Die Übertragung der Aufgaben der Koordinierungsstelle auf eine private Stiftung ähnelt mangels gesetzlicher Rechtsgrundlage einer gewillkürten Rechtsnachfolge, die nicht kraft Gesetzes zu einer Veränderung in der Zusammensetzung des Kreises der Prozessbeteiligten führt (vgl. BVerwG, Urteil v. 19. Februar 2015 - 1 C 13/14, juris, Rn. 10).

Die zulässige Verpflichtungsklage ist unbegründet. Dem Kläger steht kein Anspruch auf Akteneinsicht in die Unterlagen der Beratenden Kommission zum Fall S. zu.

Der Beklagte zu 1. hat die Akteneinsicht zu Recht verweigert, weil es sich bei ihm nicht um die für diese Entscheidung zuständige Stelle handelt. Gemäß § 7 Abs. 1 S. 1 IZG LSA entscheidet über den Antrag auf Informationszugang die Stelle nach § 1 Abs. 1 S. 1 IZG LSA, die zur Verfügung über die begehrten Informationen berechtigt ist. Von einer Verfügungsberechtigung ist auszugehen, wenn die Behörde kraft eigener Entscheidungsbefugnis den Zugang gewähren darf (vgl. LT-Drucks 5/784, S. 28). Dem Beklagten zu 1. fehlt die entsprechende Entscheidungsbefugnis im Hinblick auf die Unterlagen der Beratenden Kommission. Gemäß § 1 Abs. 3 c der Gemeinsamen Vereinbarung über die Koordinierungsstelle Magdeburg 2010-2016 nimmt die Koordinierungsstelle als unselbständige Arbeitsgruppe des Beklagten zu 1. die Funktion der Geschäftsstelle der Beratenden Kommission war. Schon aufgrund der Bezeichnung als "Geschäftsstelle" und aufgrund des Umstandes, dass insoweit die Aufgaben der Koordinierungsstelle in der Gemeinsamen Vereinbarung nicht näher bestimmt sind, ist davon auszugehen, dass es sich bei der Koordinierungsstelle um eine Einrichtung zur Entlastung und Unterstützung der Beratenden Kommission handelt, die als unregelmäßig tagendes Gremium über keinen (Verwaltungs-) Unterbau verfügt, für ihre Aufgabenerfüllung jedoch auf die Erledigung bestimmter Hilfstätigkeiten (Weiterleitung von Schreiben, Terminkoordinierung, Aktenaufbewahrung, usw.) angewiesen ist. Die Koordinierungsstelle handelt insoweit entsprechend der Vorgaben der Beratenden Kommission bzw. in Absprache mit dieser und ist daher nicht befugt, Dritten eigenmächtig Einsicht in die Akten der Beratenden Kommission zu gewähren, zumal wenn diese - wie hier - der Akteneinsicht ausdrücklich widersprochen hat. Insoweit liegt es hier anders als hinsichtlich der Eintragung und Löschung von Meldungen zu Kulturgütern auf die Internetseite www.lostart.de (vgl. § 1 Abs. 3 a der Gemeinsamen Vereinbarung über die Koordinierungsstelle Magdeburg 2010-2016), die ausschließlich nach eigenen, von der Koordinierungsstelle aufgestellten Grundsätzen erfolgt (vgl. BVerwG, Urteil v. 19. Februar 2015 - 1 C 13/14, juris, Rn. 27).
Die fehlende Entscheidungszuständigkeit der Koordinierungsstelle im vorliegenden Zusammenhang kommt auch in den an den Kläger gerichteten Schreiben vom 23. September 2013 und vom 16. Dezember 2013 zum Ausdruck. Darin lehnt nicht die Koordinierungsstelle den Antrag auf Akteneinsicht ab, sondern teilt dem Kläger die ablehnende Entscheidung der Beratenden Kommission lediglich mit.

Auch gegenüber der Beklagten zu 2. besteht kein Anspruch auf Einsicht in die Akten der Beratenden Kommission zum Fall S. Insoweit ist der Anwendungsbereich des IFG nach § 1 Abs. 1 IFG nicht eröffnet.

Gemäß § 1 Abs. 1 S. 1 IFG hat jeder nach Maßgabe dieses Gesetzes gegenüber den Behörden des Bundes einen Anspruch auf Zugang zu amtlichen Informationen. Für sonstige Bundesorgane und -einrichtungen gilt dieses Gesetz, soweit sie öffentlich-rechtliche Verwaltungsaufgaben wahrnehmen (§ 1 Abs. $1 \mathrm{~S}$. 2 IFG). Diese Voraussetzungen sind nicht erfüllt.

Es erscheint bereits zweifelhaft, ob es sich bei der Beratenden Kommission um eine Behörde, ein Organ oder eine sonstige Einrichtung des Bundes handelt, was gemäß $\S 1$ Abs. 1 S. 1 und 2 IFG Voraussetzung für die Anspruchsverpflichtung wäre. Die Beratende Kommission wurde durch eine Absprache zwischen Bund, Ländern und kommunalen Spitzenverbänden eingesetzt und ihre Mitglieder werden von der Beauftragten der Bundesregierung für Angelegenheiten der Kultur und der Medien im Einvernehmen mit der Kultusministerkonferenz und den kommunalen Spitzenverbänden berufen. Die Kommission kann im Bedarfsfall angerufen werden, wenn bei Streitigkeiten im Zusammenhang mit der Rückgabe NS-verfolgungsbedingt entzogenen Kulturgutes, insbesondere aus jüdischem Besitz, der Anspruchssteller und der über das Kulturgut Verfügende gemeinsam eine Mediation wünschen (vgl. Absprache zwischen Bund, Ländern und kommunalen Spitzenverbänden zur Einsetzung einer Beratenden Kommission im Zusammenhang mit der Rückgabe NS-verfolgungsbedingt entzogenen Kulturguts, insbesondere aus jüdischem Besitz, Beschluss der Kultusministerkonferenz v. 5. Dezember 2002). Danach dürfte es sich bei der Beratenden Kommission um ein "Mischgebilde" handeln, dass weder seiner Organisation noch seiner Funktion nach dem Bund, den Ländern oder den Kommunen eindeutig zugeordnet werden kann. Dies könnte zur Folge haben, dass weder das IFG noch ein Informationsfreiheitsgesetz der Länder - etwa das IZG LSA - auf die Beratende Kommission anwendbar und dieses Gremium damit nach keinem Informationsfreiheitsgesetz anspruchsverpflichtet wäre (vgl. Schoch, IFG, 2009, § 1 Rn. 123). Doch kann dies dahinstehen, da es sich bei der Beratenden Kommission jedenfalls um keine Bundesbehörde oder sonstige Bundeseinrichtung handelt, die öffentlich-rechtliche Verwaltungsaufgaben wahrnimmt.

Der Behördenbegriff in $\S 1$ Abs. $1 \mathrm{~S} .1$ IFG entspricht dem des $\S 1$ Abs. 4 VwVfG (BT-Drs. 15/4493, S. 7). Danach ist Behörde jede Stelle, die Aufgaben der öffentlichen Verwaltung wahrnimmt. 
Nach diesem Behördenbegriff sind informationspflichtig ohne Rücksicht auf die konkrete Bezeichnung alle vom Wechsel der in ihnen tätigen Personen unabhängigen, mit hinreichender organisatorischer Selbständigkeit ausgestatteten Einrichtungen, denen Aufgaben der öffentlichen Verwaltung und entsprechende Zuständigkeiten zur eigenverantwortlichen Wahrnehmung, d.h. zum Handeln mit Außenwirkung in eigener Zuständigkeit und im eigenen Namen, übertragen sind (vgl. Kopp/ Ramsauer, VwVfG, 15. Aufl., 2014, § 1 Rn. 51; Schoch, IFG, 2009, $\S 1 \mathrm{Rn}$. 78). Zwar ist die danach erforderliche organisatorische Selbständigkeit der Beratenden Kommission gegeben. Dieses Gremium ist - trotz der Berufung seiner Mitglieder durch die Beauftragte der Bundesregierung für Angelegenheiten der Kultur und der Medien - in keine Behörde oder sonstige öffentliche Einrichtung organisatorisch eingegliedert und es agiert autonom, d.h. außerhalb des die Verwaltungshierarchie prägenden Weisungsverhältnisses. Die Beratende Kommission übt auch keine beratende Funktion gegenüber der Verwaltung aus und bereitet auch keine Verwaltungsentscheidungen vor, wiewohl der Informationsanspruch in einem solchen Fall wohl gegenüber der beratenen Behörde geltend gemacht werden müsste (vgl. OVG NRW, Urteil v. 2. November 2010 - 8 A 475/10, juris, Rn. 56 f. mwN).

Es fehlt jedoch am außenwirksamen Handeln der Beratenden Kommission. Hierunter fällt die Befugnis zum Erlass von Verwaltungsakten, zum Abschluss öffentlich-rechtlicher Verträge im eigenen Namen oder zu sonstigem, nach öffentlichem Recht zu beurteilendem (z. B. schlicht-hoheitlichem) Handeln (vgl. Kopp/Ramsauer, VwVfG, 15. Aufl., 2014, § 1 Rn. 52). Die Tätigkeit der Beratenden Kommission beruht jedoch nicht auf Rechtssätzen des öffentlichen Rechts und bemisst sich auch nicht daran; die Abwägungsentscheidungen sind der Funktion dieses Gremiums entsprechend nicht rechtlich gebunden. Bei der Beratenden Kommission handelt es sich um ein reines Beratungsgremium von ehrenamtlich tätig werdenden, hochrangigen Persönlichkeiten aus der Wissenschaft und dem öffentlichen Leben, das bei Differenzen über die Rückgabe von Kulturgütern angerufen werden kann, die im Dritten Reich ihren Eigentümern, insbesondere verfolgten Bürgern, entzogen wurden und sich heute in Museen, Bibliotheken, Archiven oder anderen öffentlichen Einrichtungen der Bundesrepublik Deutschland befinden. Die Kommission übernimmt eine Mediatorenrolle zwischen den Trägern der Sammlungen und den ehemaligen Eigentümern der Kulturgüter bzw. deren Erben, wenn dies von beiden Seiten gewünscht wird. Zur Beilegung der Meinungsverschiedenheiten kann sie Empfehlungen gegenüber den Beteiligten aussprechen (vgl. Absprache zwischen Bund, Ländern und kommunalen Spitzenverbänden zur Einsetzung einer Beratenden Kommission im Zusammenhang mit der Rückgabe NS-verfolgungsbedingt entzogenen Kulturguts, insbesondere aus jüdischem Besitz, Beschluss der Kultusministerkonferenz v. 5. Dezember 2002).
Die Empfehlungen der Beratenden Kommission basieren auf ethischen Abwägungsentscheidungen, denen moralische Erwägungen zugrunde liegen. Rechtsnormen stellen damit keine verbindliche Grundlage für die Entscheidungen der Beratenden Kommission dar. Zwar mögen Rechtsnormen oder die ihnen zugrunde liegenden Werturteile in die Abwägung einfließen. Maßgeblich ist jedoch, dass das (öffentliche) Recht die Abwägungsentscheidungen und die darauf beruhenden Empfehlungen nicht präjudiziert, sondern nur insoweit in die Abwägung einfließt, wie die Beratende Kommission dies aus ethischen Gründen für angemessen hält. Dementsprechend könnten die Empfehlungen der Beratenden Kommission nicht nach (öffentlichem) Recht beurteilt werden, sondern ausschließlich nach den ethischen Maßstäben, auf denen sie beruhen. Insoweit spielt für die Empfehlungen der Beratenden Kommission eine maßgebliche Rolle, ob es sich um einen NS-verfolgungsbedingten Vermögensverlust gehandelt hat (vgl. etwa die Empfehlung der Beratenden Kommission in der Sache „Ba. ./. Hessen“, Pressemitteilung des Presse- und Informationsamtes der Bundesregierung Nr. 210/08 vom 12. Juni 2008, sowie in der Sache „Be. ./. Düsseldorf", Pressemitteilung vom 3. Februar 2015). Es kann aber auch darauf ankommen, ob die Direktorin eines Museums beim Erwerb eines Bildes im Hinblick auf dessen Herkunft bösgläubig gewesen ist (vgl. die Empfehlung in der Sache „W. ./. Neuss", Pressemitteilung vom 28. März 2013) oder ob entzogene Kunstwerke nach dem deutlich zum Ausdruck gebrachten Willen des Sammlers in einem Museum verbleiben sollten (vgl. die Empfehlung in der Sache "S. ./. Deutsches Historisches Museum", Pressemitteilung des Presse- und Informationsamtes der Bundesregierung vom 25. Januar 2007).

Die Beratende Kommission gibt ihre Empfehlungen ab auf der Grundlage der auf der Washingtoner Konferenz über Vermögenswerte aus der Zeit des Holocaust im Dezember 1998 verabschiedeten "Grundsätze im Bezug auf Kunstwerke, die von den Nationalsozialisten beschlagnahmt wurden" (sog. Washingtoner Erklärung). Darin haben sich die Teilnehmerstaaten, darunter die Bundesrepublik Deutschland, verpflichtet, Kunstwerke, die während der Zeit des Nationalsozialismus beschlagnahmt wurden, ausfindig zu machen, die rechtmäßigen Eigentümer oder deren Erben zu finden und rasch die notwendigen Schritte zu unternehmen, um zu „fairen und gerechten“ Lösungen zu gelangen. Im Dezember 1999 hat die Kultusministerkonferenz eine gemeinsame politische Grundsatzerklärung der Bundesregierung, der Länder und der kommunalen Spitzenverbände zur Auffindung und zur Rückgabe NS-verfolgungsbedingt entzogenen Kulturgutes, insbesondere aus jüdischem Besitz, verabschiedet (Beschluss der Kultusministerkonferenz v. 9. Dezember 1999). Hier wird im Sinne der Washingtoner Erklärung erneut die Bereitschaft zum Ausdruck gebracht, unter nationalsozialistischer Herrschaft enteignete oder geraubte Kulturgüter in öffentlichen Archiven, Museen und Bibliotheken zu suchen und faire Lösungen für die Rückgabe oder Entschädigung früherer Eigentümer bzw. deren Erben zu finden. In einem weiteren Schritt wurde im Dezember 2002 nach eingehenden Erörterungen mit den an der Verabschiedung der "Gemeinsamen Erklä- 
rung" vom 9. Dezember 1999 beteiligten Ebenen (Beauftragte der Bundesregierung für Kultur und Medien, Kultusministerkonferenz, kommunale Spitzenverbände) beschlossen, die Beratende Kommission einzurichten, die besonders problematische Fälle von Rückgaben beratend begleiten soll. Der Beratenden Kommission kommt - wie ausgeführt - vor allem die Aufgabe zu, dem Anspruchsteller und den über das Kulturgut Verfügenden für eine Mediation zur Verfügung zu stehen.

Die Washingtoner Erklärung, die mit der Maßgabe "fairer und gerechter Lösungen“ den Fixpunkt für die ethischen Abwägungsentscheidungen der Beratenden Kommission bildet, ist allerdings nur eine rechtlich unverbindliche Absichtserklärung, die folglich auch nicht nach Art. 59 Abs. 2 GG in Bundesrecht transformiert wurde. Rechtlich gleichermaßen unverbindlich sind die Gemeinsame Erklärung der Bundesregierung, der Länder und der kommunalen Spitzenverbände vom 9. Dezember 1999 (vgl. BVerwG, Urteil v. 24. November 2011 - 7 C 12/10, juris, Rn. 43; vgl. auch OVG LSA, Urteil v. 23. Oktober 2013 - 3 L 84/12, juris, Rn. 39). Die Tätigkeit der Beratenden Kommission entspricht damit zwar einem politischen Ziel der Bundesrepublik Deutschland, welches sich jedoch nicht rechtlich verfestigt hat und damit auch nicht als (öffentlich-)rechtliche Grundlage für die Empfehlungen der Beratenden Kommission anzusehen ist.

Dass sich die Tätigkeit der Beratenden Kommission nicht nach öffentlichem Recht bemisst, zeigt sich auch daran, dass ihren Empfehlungen die Rechtsverbindlichkeit und auch jegliche sonstige Rechtswirksamkeit fehlt, die Kennzeichen außenwirksamen Handelns ist. Die Beratende Kommission wird nur im Einverständnis der Beteiligten im Wege der Mediation tätig; ihre Empfehlungen sind für die Beteiligten nicht verbindlich. Ob die Beteiligten den Empfehlungen der Kommission folgen, entscheiden sie ausschließlich selbst. Die Empfehlungen der Beratenden Kommission sind für sich ggf. anschließende Rechtsstreitigkeiten auch nicht vorgreiflich oder sonst relevant, wie gerade der Fall des Herrn P. S. zeigt, der seinen Rechtsanspruch auf Herausgabe der Plakatsammlung erfolgreich auf dem Rechtsweg durchgesetzt hat. Dies unterscheidet die Empfehlungen der Beratenden Kommission von Empfehlungen anderer beratender Gremien oder Ausschüsse, die in Bereichen, in denen keine näheren rechtlichen Vorgaben bestehen, die herrschende Verkehrsauffassung beschreiben oder wegen ihrer Leitbildfunktion prägend und korrigierend auf den Handelsbrauch einwirken und dadurch im Rechtsverkehr eine große - faktische - Bedeutung erlangen können (zu einer solchen Konstellation vgl. OVG NRW, Urteil v. 2. November 2010 - 8 A 475/10, juris, Rn. 75 ff.).

Soweit der Kläger die Behördeneigenschaft der Beratenden Kommission aus der Finanzierung dieses Gremiums durch öffentliche Haushaltsmittel ableitet, verkennt er, dass durch Haushaltsmittel auch Bereiche außerhalb der staatlichen Verwaltung finanziert werden können, z.B. durch Zuwendungen ( $\$ 23 \mathrm{BHO}$ ) oder Aufwendungsersatz (§ 91 Abs. 1 S. 1 Nr. 1 BHO).
Fehlt es am außenwirksamen Handeln der Beratenden Kommission, kommt auch ein Informationsanspruch nach § 1 Abs. $1 \mathrm{~S}$. 2 IFG nicht in Betracht, weil dieser Anspruch die Wahrnehmung öffentlich-rechtlicher Verwaltungsaufgaben durch sonstige Bundesorgane und -einrichtungen voraussetzt. Danach muss auch die Tätigkeit eines sonstigen Bundesorgans oder einer sonstigen Bundeseinrichtung ihre Grundlage im öffentlichen Recht haben (vgl. Schoch, IFG, 2009, § 1 Rn. 93), woran es - wie ausgeführt - hier fehlt. Insoweit kommt hinzu, dass nach dem Willen des Gesetzgebers unabhängige Tätigkeiten wie die Rechtsprechung, Gesetzgebung oder geld- und währungspolitische Beratungen der Deutschen Bundesbank vom Informationszugang ausgenommen bleiben sollen (vgl. BT-Drs. 15/4493, S. 8). Auch die Beratende Kommission ist in ihren Empfehlungen unabhängig und damit nach der Ratio von $\S 1$ Abs. 1 S. 2 IFG nicht auskunftsverpflichtet.

Schließlich lässt sich der Anspruch auch nicht auf § 1 Abs. $1 \mathrm{~S}$. 3 IFG stützen. Danach steht einer Behörde eine natürliche oder juristische Person des Privatrechts gleich, soweit eine Behörde sich dieser Person zur Erfüllung ihrer öffentlich-rechtlichen Aufgaben bedient. Paradebeispiel hierfür ist der Verwaltungshelfer (vgl. BT-Drs. 15/4493, S. 8), doch erschöpft sich der Anwendungsbereich der Vorschrift darin nicht. Entscheidend ist, dass die durch den Privaten wahrgenommene Aufgabe - wie bei $\S 1$ Abs. 1 S. 2 IFG - im öffentlichen Recht wurzelt, d.h. durch das öffentliche Recht begründet ist (vgl. Schoch, IFG, 2009, § 1 Rn. 93). Daran fehlt es, weil die Beratende Kommission - wie ausgeführt - nicht auf der Grundlage des öffentlichen Rechts handelt. Im Übrigen wäre der Anspruch auf Informationszugang insoweit nicht gegen die Beratende Kommission oder deren Mitglieder als Private zu richten, sondern gegen die Behörde, deren Aufgaben der Antrag betrifft (vgl. BT-Drs. 15/4493, S. 8).

Da die Beratende Kommission gemäß § 1 Abs. 1 IFG bereits dem Grunde nach nicht auskunftsverpflichtet ist, muss nicht entschieden werden, ob und ggf. in welchem Umfang vorliegend der Ausschlussgrund des Schutzes der Vertraulichkeit von Verhandlungen und Beratungen von Behörden gemäß $\S 3 \mathrm{Nr} .3$ b IFG einschlägig wäre (vgl. hierzu OVG NRW, Urteil v. 2. November 2010 - 8 A 475/10, juris, Rn. 82 ff.).

Die Berufung war wegen grundsätzlicher Bedeutung der Rechtssache zuzulassen ( $\S 124$ a Abs. 1 S. 1, § 124 Abs. 2 Nr. 3 VwGO). (Entscheidung von der Redaktion bearbeitet.) 